\title{
Acute Kidney Injury After Cardiac Surgery by Complete KDIGO Criteria Predicts Increased Mortality
}

\section{Petaja, Liisa}

2017-06

Petaja , L , Vaara , S , Liuhanen , S , Suojaranta-Ylinen, R , Mildh , L , Nisula , S , Korhonen , A-M , Kaukonen, K-M , Salmenpera, M \& Pettila , V 2017 , ' Acute Kidney Injury After Cardiac Surgery by Complete KDIGO Criteria Predicts Increased Mortality ' , Journal of Cardiothoracic and Vascular Anesthesia , vol. 31 , no. 3 , pp. 827-836 . https://doi.org/10.1053/j.jvca.2016.08.026

http://hdl.handle.net/10138/297842

https://doi.org/10.1053/j.jvca.2016.08.026

publishedVersion

Downloaded from Helda, University of Helsinki institutional repository.

This is an electronic reprint of the original article.

This reprint may differ from the original in pagination and typographic detail.

Please cite the original version. 


\title{
Acute Kidney Injury After Cardiac Surgery by Complete KDIGO Criteria Predicts Increased Mortality
}

\author{
Liisa Petäjä, $\mathrm{MD}^{*, 1}$, Suvi Vaara, MD, $\mathrm{PhD}^{\dagger}$, Sasu Liuhanen, MD, BSc*, \\ Raili Suojaranta-Ylinen, $\mathrm{MD}, \mathrm{PhD}^{*}$, Leena Mildh, $\mathrm{MD}, \mathrm{PhD}^{\dagger}$, \\ Sara Nisula, $\mathrm{MD}, \mathrm{PhD}^{\dagger}$, Anna-Maija Korhonen, $\mathrm{MD}, \mathrm{PhD}^{\dagger}$, \\ Kirsi-Maija Kaukonen, $\mathrm{MD}, \mathrm{PhD}^{\dagger}$, Markku Salmenperä, $\mathrm{MD}, \mathrm{PhD}^{*}$, \\ Ville Pettilä, $\mathrm{MD}, \mathrm{PhD}^{\dagger}$ \\ *Division of Anesthesiology, Helsinki University and Helsinki University Hospital, Helsinki, Finland \\ ${ }^{\dagger}$ Division of Intensive Care, Department of Anesthesiology, Intensive Care and Pain Medicine, \\ Helsinki University and Helsinki University Hospital, Helsinki, Finland
}

Objectives: Acute kidney injury (AKI) occurs frequently after cardiac surgery and is associated with increased mortality. The Kidney Disease: Improving Global Outcomes (KDIGO) criteria for diagnosing AKI include creatinine and urine output values. However, the value of the latter is debated. The authors aimed to evaluate the incidence of AKI after cardiac surgery and the independent association of KDIGO criteria, especially the urine output criterion, and 2.5-year mortality.

Design: Prospective, observational, cohort study.

Setting: Single-center study in a university hospital.

Participants: The study comprised 638 cardiac surgical patients from September 1, 2011, to June 20, 2012.

Interventions: None.

Measurements and Main Results: Hourly urine output, daily plasma creatinine, risk factors for AKI, and variables for EuroSCORE II were recorded. AKI occurred in $183(28.7 \%)$ patients. Patients with AKI diagnosed using only urine output had higher 2.5-year mortality than did patients without AKI (9/53 [17.0\%] $v 23 / 455$ [5.1\%], $\mathrm{p}=0.001)$. AKI was associated with mortality (hazard ratios [95\% confidence intervals]: 3.3 [1.8-6.1] for KDIGO 1; 5.8 [2.7-12.1] for KDIGO 2; and 7.9 [3.5-17.6]) for KDIGO 3. KDIGO stages and AKI diagnosed using urine output were associated with mortality even after adjusting for mortality risk assessed using EuroSCORE II and risk factors for AKI.

Conclusions: AKI diagnosed using only the urine output criterion without fulfilling the creatinine criterion and all stages of AKI were associated with long-term mortality. Preoperatively assessed mortality risk using EuroSCORE II did not predict this AKI-associated mortality.

(c) 2017 Elsevier Inc. All rights reserved.

Key Words: cardiac surgery; acute kidney injury; mortality; EuroSCORE II

ACUTE KIDNEY INJURY (AKI) is associated with increased mortality and morbidity. ${ }^{1}$ Of cardiac surgical

\footnotetext{
${ }^{1}$ Address reprint requests to Liisa Petäjä, MD, Division of Anesthesiology, Department of Anesthesiology, Intensive Care and Pain Medicine, Helsinki University and Helsinki University Hospital, PO Box 340, FIN-00029 HUS, Helsinki, Finland.

E-mail address: liisa.petaja@hus.fi (L. Petäjä).
}

patients, $25 \%$ to $30 \%$ develop $\mathrm{AKI}^{2}$ Recently, the Kidney Disease: Improving Global Outcomes (KDIGO) committee published new guidelines for diagnosing and staging AKI to replace the Risk, Injury, Failure, Loss of function, End-stage renal disease and the Acute Kidney Injury Network criteria. ${ }^{3}$ All 3 definitions are based on changes in serum creatinine and/ or urine output (UO), which serve as surrogates for glomerular filtration rate. 
Although renal function in cardiac surgery has been studied extensively, only few investigators have used complete criteria with UO. ${ }^{4-6}$ UO has been suggested to be too sensitive and influenced by factors other than AKI, such as volume status and diuretics, antidiuretic hormone, and angiotensin II. $^{7,8}$ Recently, among general intensive care unit (ICU) patients, AKI diagnosed using the UO criterion alone has been associated with adverse long-term outcome. ${ }^{9}$ Previous results of the association between oliguria and postoperative survival after cardiac surgery are conflicting. 5,6

Postoperative AKI based on the KDIGO classification was an independent predictor of 30-day mortality after cardiac surgery, ${ }^{10}$ and patients with even mild AKI had increased mortality compared with their propensity-matched pairs without AKI. ${ }^{11}$ However, those authors used only the creatinine criteria in the AKI diagnosis.

Accordingly, the authors of the study presented here aimed to study the following: (1) the incidence of AKI using the complete KDIGO criteria, including UO; (2) the association between AKI and especially the UO criterion and long-term survival; and (3) whether KDIGO stages are associated with mortality independently of EuroSCORE II and known risk factors for AKI.

\section{Methods}

\section{Patients}

This single-center FINNAKI-HEART study was performed alongside the multicenter FINNAKI-study in the Helsinki University Hospital. ${ }^{12}$ The Research Ethics Committee of the Department of Surgery in Helsinki University Hospital gave approval for the study and for a deferred consent policy. Informed consent was obtained from all patients or their nextof-kin before the data were used.

The study consisted of the 2 following cohorts of cardiac surgical patients admitted to the ICU: consecutive coronary artery bypass grafting patients (CABG) from September 1, 2011, to June 20, 2012, and consecutive other cardiac surgical patients, for a shorter period, from September 1, 2011, to January 31, 2012. Predefined exclusion criteria were as follows: transplantations and implantations of ventricular assist devices, refusal of transfusions, noncitizens, no informed consent, and transfemoral valve implantations. Patients on dialysis before surgery and patients without complete data on fluid therapy also were excluded.

\section{Data Collection}

Routine data, including demographics, physiologic data, hourly UO, ICU length of stay (LOS), and ICU and hospital mortality were recorded prospectively in the Finnish Intensive Care Consortium database (Tieto Ltd, Helsinki, Finland). Calculations of AKI stage based on UO were performed automatically by a calculator (built in the database for the FINNAKI main study). UO was measured hourly in the ICU, and the calculator considered each measurement. If oliguria lasted more than 6,12 , or 24 hours in the ICU, the corresponding stage of AKI, according to KDIGO criteria, was recorded for the patient. For each patient, the first UOAKI and the highest UO-AKI were recorded during the first 5 days of ICU stay.

Mortality data were obtained within 3 years for the main FINNAKI cohort from the Population Register Centre (http:// www.vrk.fi). FINNAKI heart patients were followed up for approximately 2.5 years (961 days).

Perioperative and postoperative treatment, type of fluid given, and daily fluid balance were recorded prospectively in an electronic anesthesia and intensive care patient data management system (Picis, Wakefield, MA, and Dräger Medical, Lübeck, Germany). A study-specific case report form was completed at admission and daily until discharge from the ICU until the fourth postoperative day. The form included baseline creatinine value, patients' chronic illnesses, medication, and daily total fluid balance.

Plasma creatinine was measured daily and UO registered hourly during the ICU stay. After ICU discharge and until the fourth postoperative day, creatinine values were obtained according to the decision of the clinician.

\section{Definitions}

AKI was defined and staged according to the KDIGO guidelines $^{3}$ and considered creatinine (Crea-AKI), UO (UO-AKI), both creatinine and UO (Crea+UO-AKI), and renal replacement therapy during the ICU stay; after ICU discharge and until the fourth postoperative day, only creatinine and renal replacement therapy (RRT) were used. The development of AKI during hospitalization before surgery also was measured. Baseline creatinine was defined as the latest value from the previous year prior to ICU admission, excluding the preceding seven days, as suggested for ICU patients. Thus, the AKI developed perioperatively, covering also the immediate hospitalization before surgery. If the baseline value was not available, the baseline creatinine was estimated using the Modification of Diet in Renal Disease (MDRD) formula, which assumes a glomerular filtration rate of $75 \mathrm{~mL} / \mathrm{min} /$ $1.73 \mathrm{~m}^{2}{ }^{13}$ To cover the acute increase of creatinine in the KDIGO definition $(0.3 \mathrm{mg} / \mathrm{dL}$ in 48 hours), daily creatinine also was compared with creatinine values of the 2 previous days. The first and the highest stages of AKI were recorded for each patient over 5 days, including the day of surgery.

The vasopressor load was defined using the vasoactive dose as the norepinephrine-equivalent dose. ${ }^{14-16}$ The variable was completed by adding ephedrine ${ }^{17}$ and vasopressin doses. ${ }^{15}$ The vasopressor load was as follows: $(\mu \mathrm{g} / \mathrm{kg} / \mathrm{min})=$ norepinephrine $(\mu \mathrm{g} / \mathrm{kg} / \mathrm{min})+$ dopamine $(\mu \mathrm{g} / \mathrm{kg} / \mathrm{min}) / 4+$ epinephrine $(\mu \mathrm{g} /$ $\mathrm{kg} / \mathrm{min})+$ phenylephrine $(\mu \mathrm{g} / \mathrm{kg} / \mathrm{min}) / 10+$ ephedrine $(\mu \mathrm{g} / \mathrm{kg} /$ $\mathrm{min}) / 800+$ vasopressin $(\mathrm{IU} / \mathrm{kg} / \mathrm{min}) \times 500$

The vasopressor load was calculated separately for operating room time and daily in the ICU. Inotropes milrinone, levosimendan, and dobutamine were combined as another variable, inodilator therapy. 


\section{Anesthesia and Surgery}

In the authors' institution, anesthesia is induced with propofol or etomidate; opioids (fentanyl, alfentanil, or sufentanil); and rocuronium. Anesthesia is maintained with opioid infusion and sevoflurane. At the end of surgery, propofol infusion is started for sedation during the first hours in the ICU. Surgery is performed mostly on-pump at tepid temperature, but occasionally by off-pump technique or, rarely, in deep hypothermia in case of aortic arch correction. Surgical incision mainly is by sternotomy but occasionally by ministernotomy, lateral thoracotomy, or thoracoscopy in case of robotic surgery. Cannulation sites are the ascending aorta or femoral artery and right auricle, bicaval, or femoral vein, when appropriate. No mannitol is given, and perioperative furosemide is administered only occasionally. The heart is protected with intermittent cold blood cardioplegia.

\section{Statistical Analyses}

Variables with skewed distribution are expressed as medians and interquartile ranges (IQR). Comparisons of continuous variables were made using the Mann-Whitney U-test. Frequencies were compared using the chi-square or Fisher's exact test, when appropriate. A 2-sided $\mathrm{p}$ value $<0.05$ was considered significant. Only risk factors present until the day preceding the diagnosis of AKI or risk factors present in the operating room for patients developing AKI on the day of surgery were considered. The median for time preceding AKI was calculated and used as the corresponding period for patients without AKI. ${ }^{18}$

The power calculation was performed according to a primary endpoint of the FINNAKI-HEART study and was published previously. ${ }^{19}$ AKI was a secondary preplanned endpoint. This study's sample of more than 600 cardiac surgical patients, which was estimated to include 150 to 200 cases of AKI, was assessed as being adequate to draw conclusions regarding the incidence, severity, and baseline characteristics of these patients.

A multivariable logistic regression analysis was performed to explore the independence of the risk factors for AKI. From 8 prediction models for AKI after cardiac surgery, ${ }^{20}$ all variables that were in more than 1 model were included, and the analysis was supplemented by medication; vasopressor load; and use of inodilators, diuretics, colloids, and blood products. The number of covariates was restricted to 1 per 8 dependent endpoints ${ }^{21}$ and covariates with the strongest association with AKI were taken into the multivariable analyses. Analyses were performed by entering all of the covariates in the model at the same time without any stepwise approach. Interactions between the variables of the lowest perioperative hematocrit value and red blood cell transfusions were excluded by introducing an interaction term in the analysis. Model goodness of fit was tested using the Hosmer-Lemeshow test and C statistics. A separate multivariable model with the same principles was created for UOAKI patients only.
This study presents the 2.5-year mortality of patients with AKI diagnosed using different methods (UO, creatinine, or both) and patients without AKI as Kaplan-Meier curves and tested their difference using the Breslow test. In addition, Cox regression was used to evaluate the association between these groups and the postoperative KDIGO stages and mortality. The results were adjusted, first by EuroSCORE II to explore whether KDIGO stages were associated with mortality independently and second by EuroSCORE II and risk factors for AKI. Because EuroSCORE II includes many risk factors for postoperative AKI, only those risk factors that were associated independently with postoperative AKI in logistic regression but not in EuroSCORE II were included in the second analysis. Interaction between EuroSCORE II and KDIGO stages was excluded by interaction term in the analysis. Analysis was performed using the enter method. Proportionality was confirmed by visual inspection of the Kaplan-Meier curves.

Four sensitivity analyses were performed and excluded the following: (1) patients whose baseline creatinine value was estimated, (2) patients with chronic kidney disease, (3) patients who underwent surgery after January 31 (after that, only CABG patients were enrolled), and (4) patients who underwent surgery without cardiopulmonary bypass. In addition, stratified analyses of mortality in the Crea-AKI, UO-AKI, and Crea + UO-AKI groups were performed separately for patients who did and did not receive furosemide postoperatively. Furthermore, mortality analyses using Kaplan-Meier curves were repeated and excluded UO-AKI and Crea+UO-AKI patients who received their diagnoses after 24 postoperative hours in an effort to eliminate the possible detection bias caused by patients discharged from the ICU after 24 hours during which time UO data were no longer being recorded.

All analyses were performed using IBM SPSS Statistics, Version 22 (IBM Corp, Armonk, NY).

\section{Results}

\section{Surgery}

The study comprised 638 cardiac surgical patients (Fig 1). Of those $638,420(65.8 \%)$ underwent coronary surgery, 141 $(22.1 \%)$ valve surgery, $51(8.0 \%)$ coronary and valve surgery, and $26(4.1 \%)$ other cardiac surgery. Sixty of the $638(9.4 \%)$ patients underwent surgery without cardiopulmonary bypass.

\section{Incidence of AKI}

AKI was diagnosed in 183 (28.7\%; $95 \%$ confidence interval $25.3 \%-32.3 \%$ ) patients. There was no difference in the incidence of AKI between the patients who underwent surgery before January 31 and those after that-128/445 (28.8\%) versus 55/138 (28.5\%). Eighteen (2.8\%) patients received RRT within 5 days, and 1 patient remained on chronic dialysis at the time of hospital discharge.

Of the 183 AKI patients, 53 (29.0\%) were diagnosed using only the UO criterion, 45 of whom experienced stage 1 and 


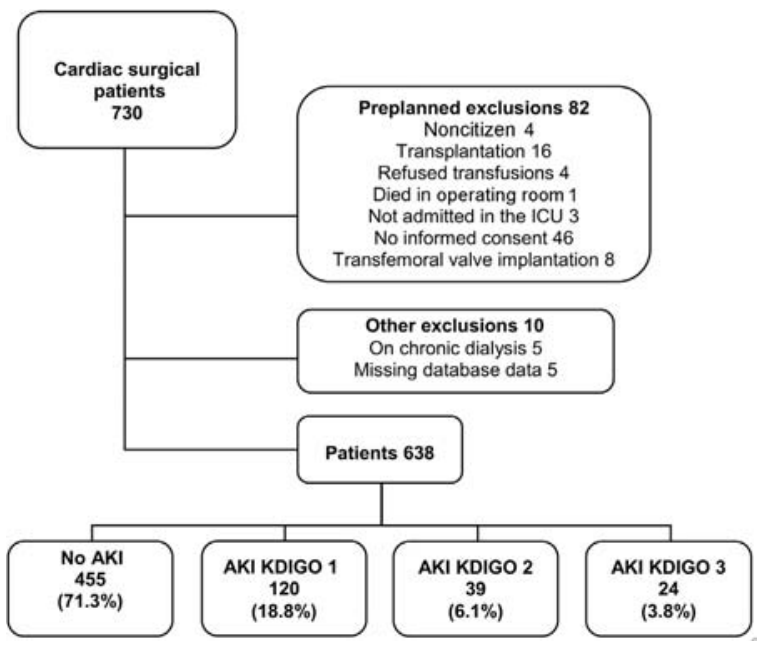

Fig 1. Study flow chart.

8 of whom experienced stage 2 AKI. Another 76 (41.5\%) AKI patients were diagnosed using only the creatinine criterion, and 36 (19.7\%) AKI patients were diagnosed using both the UO and creatinine criteria. For 199 (31.2\%) patients, the baseline creatinine value had to be estimated using the MDRD formula. The mean and median of the estimated creatinine clearance using the MDRD formula for those whose baseline creatinine data were available were 77.7 (95\% confidence interval 75.680.0 ) $\mathrm{mL} / \mathrm{min} / 1.73 \mathrm{~m}^{2}$ and 76.1 (IQR $61.9-90.8$ ) $\mathrm{mL} / \mathrm{min} / 1.73$ $\mathrm{m}^{2}$, respectively.

\section{Timing of AKI}

AKI was diagnosed in the ICU in 137 (74.9\%) of 183 patients and in the ward (based on the creatinine value) in 46 (25.1\%) patients. KDIGO AKI stages and criteria are summarized in Table 1. The median (interquartile range [IQR]) postoperative day for the first AKI diagnosis was 1 (1-2) day. More than $80 \%$ of AKI cases were diagnosed before the third postoperative day. The daily appearance of the highest KDIGO diagnosis is presented in Figure 2.

The UO criterion was fulfilled on average (median [IQR]) in 23.5 (9.75-35.75) hours in the UO-AKI group and in 21 (9-35) hours in the UO+Crea-AKI group. Timing of the first diagnosis of UO-AKI in the UO-AKI and Crea+UO-AKI groups are presented in Supplemental Figures 1, A and B.

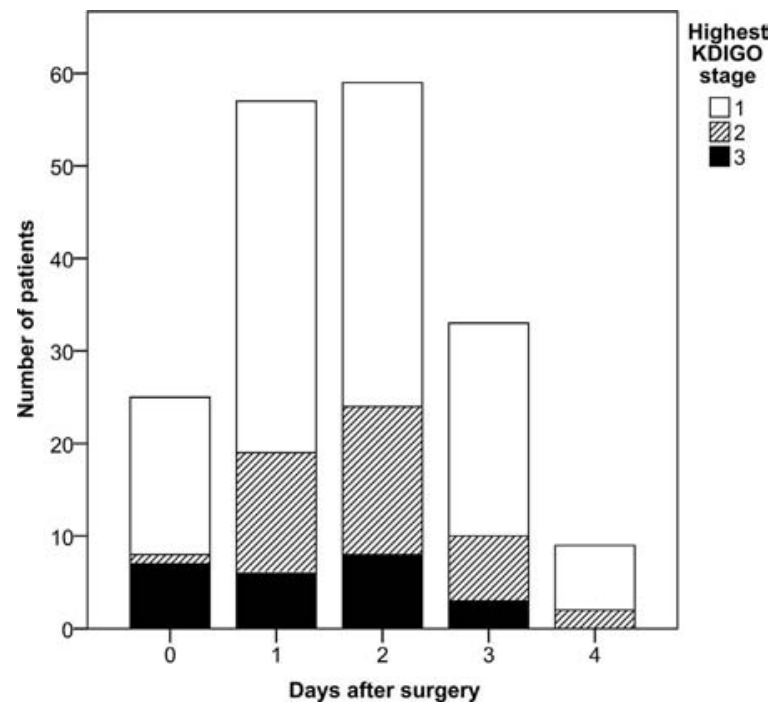

Fig 2. Daily appearance of acute kidney injury stages according to Kidney Disease: Improving Global Outcomes criteria.

UO data were complete for all patients during their ICU stay. The median (IQR) ICU LOS was 1.0 (0.9-2.1) days; in patients with no AKI the median ICU LOS was 1.0 (0.9-1.9) days, with Crea-AKI 1.1 (0.9-3.0), with UO-AKI 2.8 (1.8-3.7), and with Crea+UO-AKI 6.0 (2.0-9.7). Thus, UO data were missing in $260(40.8 \%)$ patients after 24 postoperative hours, in $360(56.4 \%)$ patients after 36 postoperative hours, and in 459 (71.9\%) patients after 48 postoperative hours (Supplemental Fig 2).

\section{Patient Characteristics and Risk Factors for AKI}

Patients with AKI received more blood products and vasoactive agents (Table 2). Age, body mass index (BMI), peripheral vascular disease, chronic kidney disease, urgent surgery, low perioperative hematocrit value, vasopressor load, use of inodilators, and postoperative furosemide were significant risk factors for AKI in multivariable logistic regression analysis (Table 3).

Few differences in demographics and intraoperative variables between the UO-AKI and Crea-AKI groups were detected; patients in the UO-AKI group received fewer vasopressors, more inodilators, and less furosemide postoperatively compared

Table 1

AKI Diagnosis According to KDIGO Criteria

\begin{tabular}{|c|c|c|c|c|c|c|c|}
\hline Patients & $\begin{array}{c}\text { Crea }^{1} \text { Criterion } \\
\text { Only }\end{array}$ & $\begin{array}{l}\text { UO Criterion } \\
\text { Only }\end{array}$ & $\begin{array}{c}\text { Crea and UO } \\
\text { Criteria }\end{array}$ & $\begin{array}{l}\text { RRT and Crea } \\
\text { Criteria }\end{array}$ & $\begin{array}{l}\text { RRT and UO } \\
\text { Criteria }\end{array}$ & $\begin{array}{l}\text { RRT, Crea, and } \\
\text { UO Criteria }\end{array}$ & $\begin{array}{c}\text { All Criteria (\% of AKI } \\
\text { patients }\end{array}$ \\
\hline Highest KDIGO 1 & 55 & 45 & 20 & 0 & 0 & 0 & $120(65.6 \%)$ \\
\hline Highest KDIGO 3 & 3 & 0 & 3 & 0 & 1 & $17^{*}$ & $24(13.1 \%)$ \\
\hline $\begin{array}{l}\text { Total amount (\% of } \\
\text { patients with AKI) }\end{array}$ & $76(46.4 \%)$ & $53(29.0 \%)$ & $36(19.7 \%)$ & $0(0.0 \%)$ & $1(0.5 \%)$ & $17(9.3 \%)$ & 183 \\
\hline
\end{tabular}

Abbreviations: crea, plasma creatinine; KDIGO, Kidney Disease: Improving Global Outcome; UO, urine output; RRT, renal replacement therapy

*Four of these patients had KDIGO stage 2 without the RRT criterion. 
Table 2

Characteristics of Patients With and Without AKI

\begin{tabular}{|c|c|c|c|}
\hline Patient Characteristic & No AKI & AKI & p Value \\
\hline Number of patients & 455 & 183 & \\
\hline Age, years & $66(59-74)$ & $72(63-78)$ & $<0.001$ \\
\hline Female & $107(23.5 \%)$ & $53(29.0 \%)$ & 0.15 \\
\hline Body mass index $>30 \mathrm{~kg} / \mathrm{m}^{2}$ & $111(24.4 \%)$ & $73(39.9 \%)$ & 0.001 \\
\hline Hypertension & $284(62.4 \%)$ & $128(69.9 \%)$ & 0.072 \\
\hline Left ventricular ejection fraction, $\leq 30 \%$ & $15(3.3 \%)$ & $12(6.6 \%)$ & 0.064 \\
\hline Peripheral vascular disease & $60(13.2 \%)$ & $45(24.6 \%)$ & $<0.001$ \\
\hline Chronic obstructive pulmonary disease & $40(8.8 \%)$ & $23(12.6 \%)$ & 0.15 \\
\hline Insulin-dependent diabetes mellitus & $36(7.9 \%)$ & $25(13.7 \%)$ & 0.026 \\
\hline Noninsulin-dependent diabetes mellitus & $81(17.8 \%)$ & $30(16.4 \%)$ & 0.67 \\
\hline Chronic kidney disease & $27(6.0 \%)$ & $43(23.9 \%)$ & $<0.001$ \\
\hline Recent acute myocardial infarction & $145(31.9 \%)$ & $65(35.5 \%)$ & 0.38 \\
\hline \multicolumn{4}{|l|}{ Medication 48 hours before admission } \\
\hline Contrast agent & $39(8.6 \%)$ & $26(14.2 \%)$ & 0.035 \\
\hline ACE-inhibitor/AT-antagonist & $194(42.9 \%)$ & $85(46.7 \%)$ & 0.39 \\
\hline Nonsteroidal anti-inflammatory drugs & $11(2.4 \%)$ & $5(2.7 \%)$ & 0.82 \\
\hline Diuretics & $103(22.8 \%)$ & $68(37.8 \%)$ & $<0.001$ \\
\hline \multicolumn{4}{|l|}{ Perioperative variables } \\
\hline Prior cardiac surgery & $14(3.1 \%)$ & $6(3.3 \%)$ & 0.89 \\
\hline Coronary surgery versus other surgery & $310(68.1 \%)$ & $111(60.7 \%)$ & 0.071 \\
\hline Urgent or emergency & $166(36.5 \%)$ & $92(50.3 \%)$ & 0.001 \\
\hline Cardiogenic shock & $5(1.1 \%)$ & $9(4.9 \%)$ & 0.003 \\
\hline Intra-aortic balloon pump & $7(1.5 \%)$ & $12(6.6 \%)$ & 0.001 \\
\hline Cardiopulmonary bypass time $>120 \mathrm{~min}$ & $119(26.2 \%)$ & $52(28.4 \%)$ & 0.56 \\
\hline Lowest hematocrit, \% & $29(26-33)$ & $27(24-31)$ & $<0.001$ \\
\hline Vasopressor load, $\mu \mathrm{g} / \mathrm{kg} / \mathrm{min}^{*}$ & $0.085(0.040-0.152)$ & $0.165(0.076-0.380)$ & $<0.001$ \\
\hline Any inodilator, $\mathrm{n}^{*}$ & $90(19.8 \%)$ & $88(48.1 \%)$ & $<0.001$ \\
\hline Milrinone & $66(14.5 \%)$ & $68(37.2 \%)$ & $<0.001$ \\
\hline Levosimedan & $26(5.7 \%)$ & $25(13.7 \%)$ & 0.001 \\
\hline Dobutamine & $1(0.2 \%)$ & $5(2.7 \%)$ & 0.009 \\
\hline \multicolumn{4}{|l|}{ Fluid therapy before AKI } \\
\hline Hydroxyethyl starch infusion & $366(80.4 \%)$ & $148(80.9 \%)$ & 0.90 \\
\hline Albumin infusion ${ }^{*}$ & $60(13.2 \%)$ & $43(23.5 \%)$ & 0.001 \\
\hline Red blood cell transfusion* & $193(42.4 \%)$ & $120(65.6 \%)$ & $<0.001$ \\
\hline Fresh frozen plasma transfusion ${ }^{*}$ & $78(17.1 \%)$ & $58(31.7 \%)$ & $<0.001$ \\
\hline Platelet transfusion, $\mathrm{n}^{*}$ & $84(18.5 \%)$ & $55(30.1 \%)$ & 0.001 \\
\hline Furosemide in $\mathrm{ICU}^{*}$ & $86(18.9 \%)$ & $96(52.5 \%)$ & $<0.001$ \\
\hline \multicolumn{4}{|l|}{ Risk of in-hospital death } \\
\hline EuroSCORE II, \% & $1.98(1.11-3.64)$ & $3.88(1.97-7.80)$ & $<0.001$ \\
\hline
\end{tabular}

NOTE. Figures are presented as median (interquartile range) or number (percentage).

Abbreviations: ACE, angiotensin-converting enzyme; AKI, acute kidney injury; AT, angiotensin.

*Risk factor was recorded during the period before AKI and corresponding median time for patients without AKI.

with patients in the Crea-AKI group (Supplemental Table 1). In a separate multivariable model for UO-AKI patients only, BMI, chronic kidney disease, low hematocrit value, inodilator therapy, and postoperative furosemide were significant variables (Supplemental Table 2).

\section{Mortality}

Crude mortality rates at $30,90,180$ days and at 2.5 years (961 days) were $2.2 \%$ (14/638), 3.4\% (22/638), $4.2 \%$ (27/ 638), and 9.4\% (60/638), respectively. Standardized mortality rates according to EuroSCORE II were 0.43 and 0.67 at days 30 and at 90 , respectively. Mortality rates were higher in patients with AKI than those without AKI (Fig 3).

\section{Mortality According to UO and Creatinine}

There were 13, 9, and 15 deaths in the Crea-AKI, UO-AKI, and Crea+UO-AKI groups, respectively. The cause of death was cardiovascular in $7(53.8 \%)$ in the Crea-AKI group, 6 $(66.7 \%)$ in UO-AKI group, and $12(80.0 \%)$ in the Crea+UOAKI group. Cancer was the cause of death in $2(15.4 \%), 1$ $(11.1 \%)$, and $1(6.7 \%)$ in these groups, respectively. Causes of death were missing in 3 patients.

AKI patients diagnosed using the UO criterion alone had higher 2.5-year mortality than did patients without AKI (9/53 [17.0\%] $v 23 / 455$ [5.1\%], $\mathrm{p}=0.001$ ). AKI patients diagnosed using only the creatinine criterion also experienced higher 2.5-year mortality compared with patients without AKI $(13 / 76[17.1 \%] \vee 23 / 455$ [5.1\%], $\mathrm{p}<0.001)$, as did patients 
Table 3

Multivariable Regression Analysis of Covariates Predicting Acute Kidney Injury During First 5 Postoperative Days ${ }^{*}+$

\begin{tabular}{llr}
\hline & $\begin{array}{c}\text { Multivariable Odds } \\
\text { Ratio (95\% CI) }\end{array}$ & p Value \\
\hline Age, years & $1.02(1.00-1.04)$ & 0.027 \\
Body mass index $>30 \mathrm{~kg} / \mathrm{m}^{2}$, yes/no & $2.99(1.87-4.78)$ & $<0.001$ \\
Peripheral vascular disease, yes/no & $1.95(1.10-3.45)$ & 0.023 \\
Chronic kidney disease, yes/no & $4.08(2.22-7.50)$ & $<0.001$ \\
Urgent/emergency versus elective surgery & $1.76(1.11-2.78)$ & 0.017 \\
Lowest hematocrit, \% & $0.95(0.92-0.99)$ & 0.009 \\
Vasopressor load, $\mu \mathrm{g} / \mathrm{kg} / \mathrm{min}^{\ddagger}$ & $3.72(1.50-9.25)$ & 0.005 \\
Inodilator therapy, yes/no & $2.49(1.54-4.02)$ & $<0.001$ \\
Furosemide in intensive care unit,' yes/no & $3.84(2.47-5.99)$ & $<0.001$ \\
\hline
\end{tabular}

*Calibration of the multivariable model: Hosmer-Lemeshow test, $\mathrm{p}=0.43$

${ }^{\dagger}$ Discrimination of the multivariable model: C statistics 0.84 (95\% CI 0.81-0.88)

${ }^{\ddagger}$ The sum of mean daily doses before acute kidney injury or corresponding period.

diagnosed using both the creatinine and UO criteria $(8 / 36$ [22.2\%] $v 23 / 455$ [5.1\%], $\mathrm{p}<0.001)$. Patients who received RRT $(\mathrm{n}=18)$ were excluded from these groups.

Including patients who received RRT, mortality among the Crea-AKI, UO-AKI, and with Crea+UO-AKI groups was higher than in those without AKI, but mortality did not differ among the AKI groups (see Fig 3). These results remained unchanged after adjusting for EuroSCORE II (Fig 4, A and B). After adjusting for independent risk factors for AKI (not included in EuroSCORE II), namely BMI, lowest perioperative hematocrit value, vasopressor load, use of inodilators, and postoperative furosemide, the results remained unchanged (Fig 4, C).

\section{KDIGO Stage and Mortality}

Mortality rates according to KDIGO stages are presented in Fig 5. All AKI stages were associated with a shorter time to

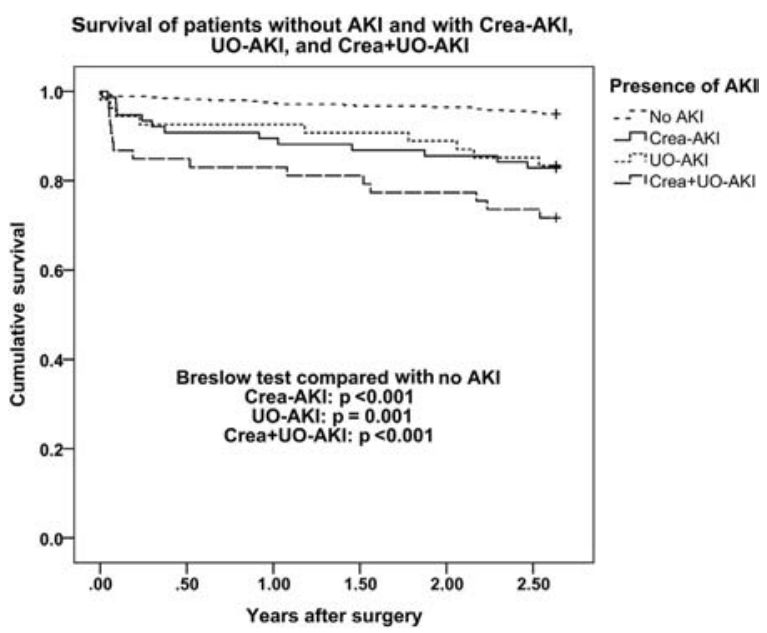

Fig 3. Kaplan-Meier survival curves of patients with acute kidney injury (AKI) diagnosed by creatinine (Crea-AKI), urine output (UO) (UO-AKI), and both creatinine and UO (Crea+UO-AKI), (18 patients who received renal replacement therapy were included). death compared with patients without AKI (see Fig 5, A). This finding remained unchanged after adjusting for EuroSCORE II (see Fig 5, B) and after adjusting for risk factors for AKI (see Fig 5, C).

\section{Sensitivity Analyses}

In sensitivity analysis 1 , which excluded patients with an estimated baseline creatinine value, the results remained unchanged. Sensitivity analyses 2 and 3 confirmed the results in all other subgroups but the Crea-AKI group, for which the last 193 CABG patients were excluded and the risk was adjusted for EuroSCORE II and additional AKI risk factors (Supplemental Table 3).

Of the 638 patients, $182(28.5 \%)$ received furosemide postoperatively in the ICU preceding AKI. Survival curves for patients who did not receive furosemide differed significantly for all AKI groups compared with those without AKI. Among patients receiving furosemide, the survival curve for the Crea+UO-AKI group differed from that of the no-AKIgroup (Supplemental Fig 3, A and B). In total, 18.9\% (86/455) of no-AKI-patients, $76.3 \%$ (58/76) of Crea-AKI patients, $38.9 \%$ (21/54) of UO-AKI patients, and $32.1 \%$ (17/53) of Crea + UO-AKI patients received diuretics.

After excluding UO-AKI and Crea+UO-AKI patients who received their diagnosis of UO-AKI after 24 postoperative hours, mortality within 961 days in all the groups were as follows: no AKI 23/455 (5.1\%), Crea-AKI 13/63 (17.1\%), UOAKI 8/28 (28.6\%), and Crea+UO-AKI 11/37 (28.9\%). KaplanMeier curves are presented in Supplemental Figure 4. Mortality was higher in all the AKI groups compared with no-AKI group.

\section{Discussion}

In this prospective, observational study, $28.7 \%$ of cardiac surgical patients presented with AKI according to KDIGO criteria. Patients with AKI diagnosed using only the KDIGO UO criterion experienced higher crude and adjusted mortality than did patients without AKI. AKI by KDIGO stages was associated with increased crude mortality, and the results remained the same after adjusting for mortality risk assessed using EuroSCORE II and other risk factors for AKI.

In this study, the incidence of AKI and the use of RRT (2.8\%) were comparable with previous reports (15\%-30\% for AKI and 1\%-5\% for RRT). ${ }^{2,22}$ Given the complete KDIGO criteria, the incidence in this study actually may have been lower than that in the literature.

Notably, patients with AKI classified using only UO, most with stage-1 AKI, experienced higher crude and adjusted long-term mortality than did patients without AKI. These patients comprised almost one-third of AKI patients, and their diagnosis would have been missed otherwise. These patients also experienced longer ICU stays than did patients without AKI. The finding of greater mortality was in line with a recent study of 32,056 ICU patients, for whom even a short episode of isolated oliguria without azotemia was associated with increased 1 -year mortality. ${ }^{9}$ In addition, the 
A

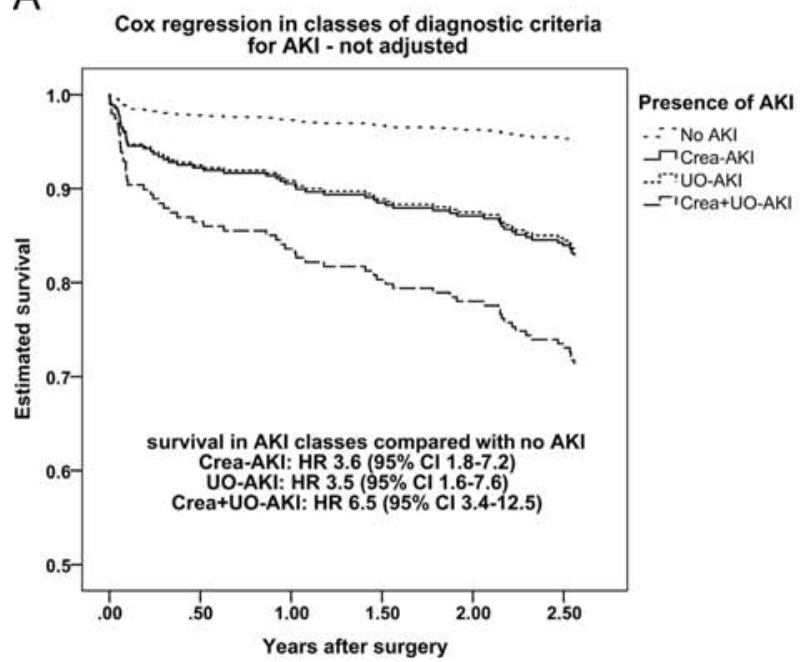

B

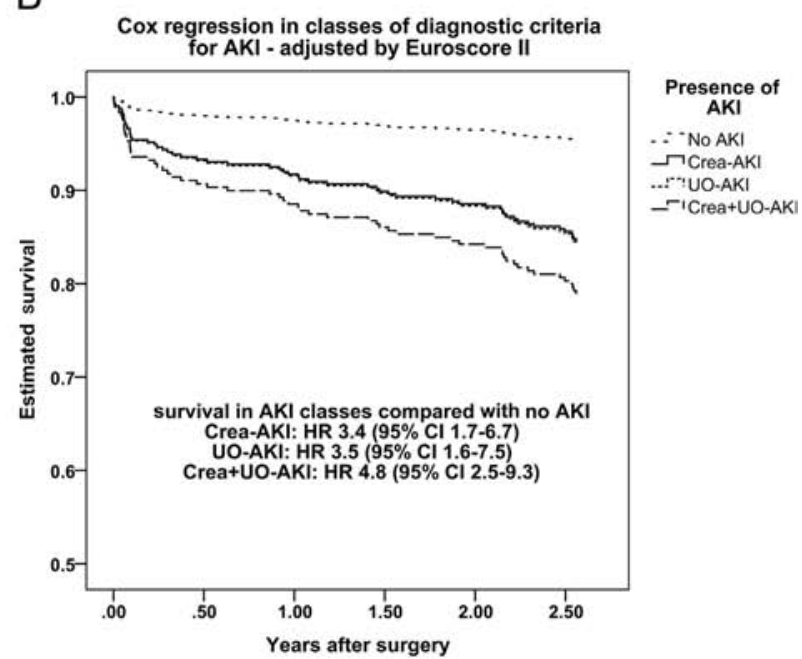

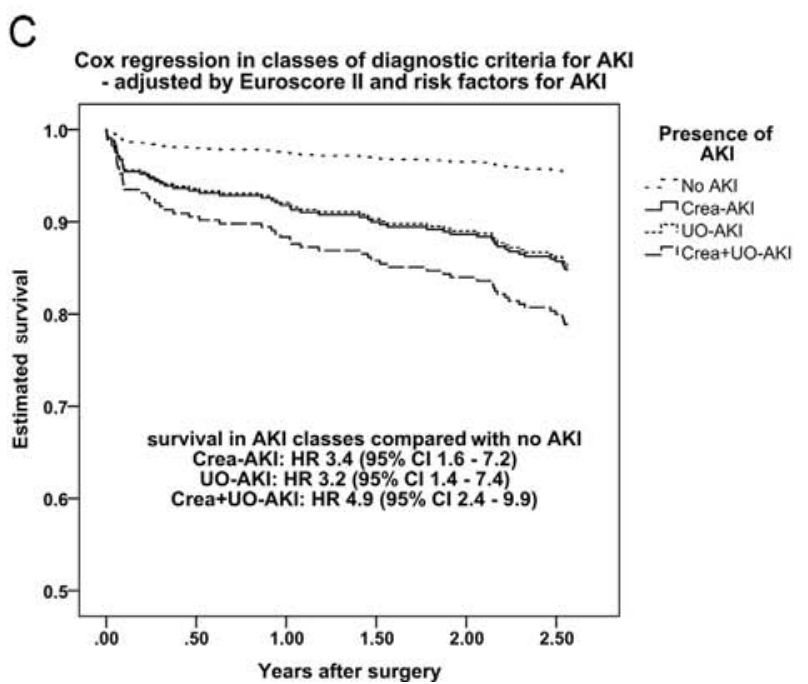

Fig 4. Cox proportional hazards model according to (A) diagnostic criteria for acute kidney injury (AKI), (B) diagnostic criteria for AKI adjusted for Eurosocre II, and (C) diagnostic criteria for AKI adjusted for EuroSCORE II and AKI risk factors (18 patients who received renal replacement therapy were included).

finding demonstrating an association of AKI diagnosed using only the UO criterion with long-term mortality highlighted the importance of including the UO criterion with the creatinine criterion in epidemiologic studies and corroborates previous reports. $^{6,9}$

Hormonal $^{23}$ and volume fluctuations occur frequently in cardiac surgery. Moreover, perioperative mannitol and postoperative diuretics may be used, which may blur the interpretation of hourly UO. In the study presented here, mannitol was not used and the use of diuretics was adjusted for in the multivariable models. Two reports from cohorts with perioperative mannitol in routine use have shown higher incidence rates for AKI, from $40 \%{ }^{5}$ to $55 \% .^{6}$ Lagny et al ${ }^{5}$ did not find oliguric AKI to be associated with increased mortality in their retrospective report including 443 coronary and/or valve surgery patients. Their use of mannitol may have affected diuresis, thus weakening UO as a diagnostic criterion for AKI. Similarly, in the study presented here, mortality among the patients who received postoperative diuretics did not differ between the UO-AKI group and patients without AKI, suggesting that the UO criterion was weaker with postoperative diuretics. McIlroy et $\mathrm{al}^{6}$ studied 311 elective on-pump coronary and/or valve surgery patients retrospectively and suggested that the UO criterion at 48 postoperative hours was too sensitive, whereas the UO criterion at 12 postoperative hours performed well for in-hospital mortality. The finding of the study presented here was in line with that by Mcllroy: Early UO-AKI was associated with mortality because nearly half of the only UO-AKI diagnoses were made within 24 hours of surgery and UO-AKI was associated with higher mortality.

Severity of AKI assessed using $\mathrm{KDIGO}^{10}$ and Acute Kidney Injury Network ${ }^{24}$ has been associated independently with mortality 30 days and 1 year after cardiac surgery, respectively. In these studies, however, the AKI diagnosis was based only on the creatinine criterion. Furthermore, the variables used to adjust the associations included fewer than half of the variables in the recently updated EuroSCORE II. A recent propensity-matched analysis reported that patients with mild AKI experienced higher mortality compared with those without AKI among 833 pairs after cardiac surgery. ${ }^{11}$ The authors 
A

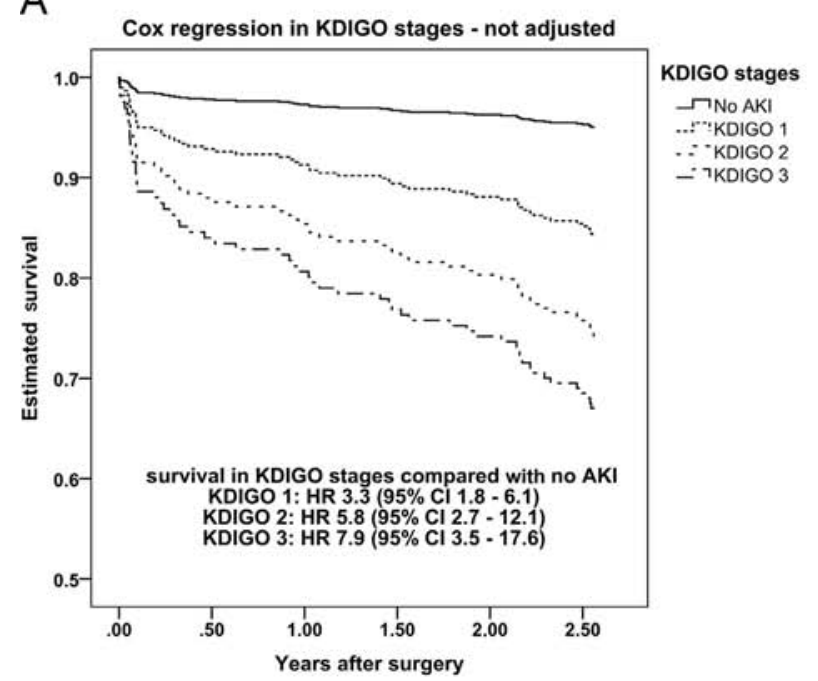

B

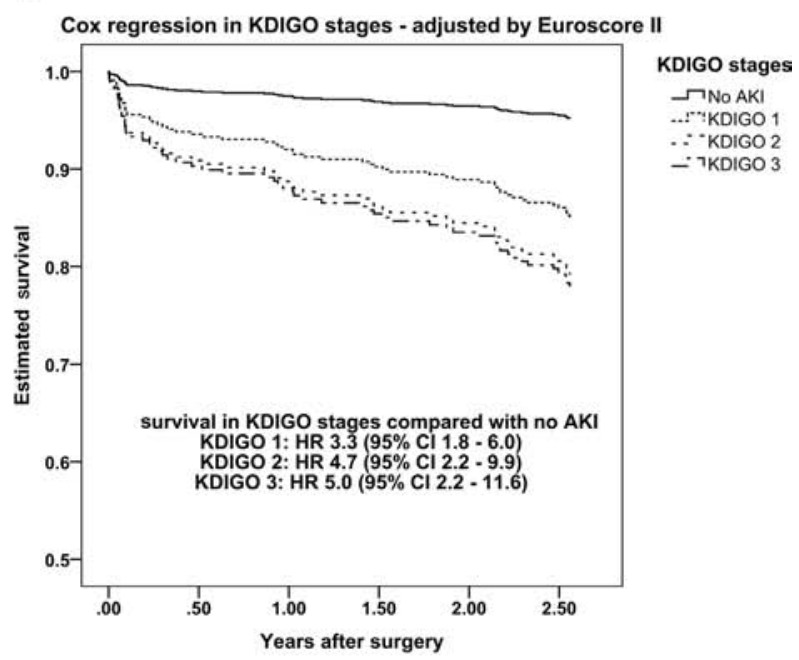

C

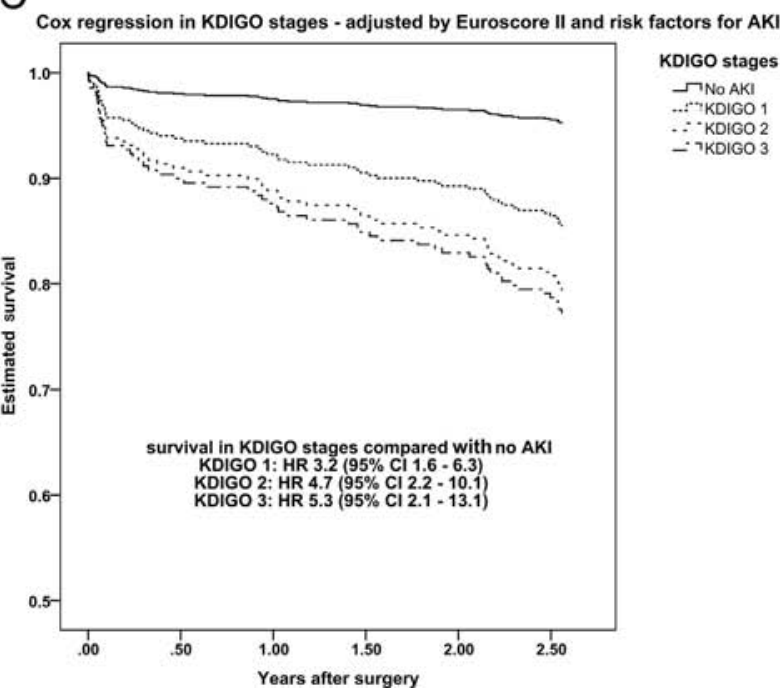

Fig 5. Cox proportional hazards model according to (A) Kidney Disease: Improving Global Outcome (KDIGO) stages, (B) KDIGO stages adjusted for EuroSCORE II, and (C) KDIGO stages adjusted for EuroSCORE II and acute kidney injury risk factors.

used several predictive variables in propensity matching but only the creatinine criterion for AKI diagnosis. Thus, the study presented here differed by using the complete KDIGO criteria and adjusting for EuroSCORE II and AKI risk factors.

The AKI risk factors detected in the study presented here, namely advanced age, high BMI, peripheral vascular disease, chronic kidney disease, and urgent surgery, have been reported consistently in previous studies. ${ }^{20}$ Low perioperative hematocrit and red blood cell transfusions both have been associated independently with AKI after cardiac surgery, ${ }^{25}$ but of these, only perioperative hematocrit was significant in the study presented here. Perioperative furosemide has been associated with mortality. ${ }^{26}$ In the study presented here, one-fourth of the patients received postoperative furosemide before AKI, and it was a risk factor for AKI. Postoperative patients receiving furosemide plausibly are those assessed to be hypervolemic, and hypervolemia per se is associated with AKI and mortality. ${ }^{27}$
In the study presented here, an association was found between vasopressor load and use of inodilators and AKI. Patients who developed AKI were more severely ill and experienced more comorbidities and a decreased left ventricular ejection fraction. Consequently, this association most likely reflected the patient characteristics rather than the use of vasopressors or inodilators. Patients in the Crea-AKI and UOAKI groups seemed to have different vasoactive agent profiles. Vasopressors were more associated with Crea-AKI patients, and inodilators were more associated with UO-AKI patients.

This study had some limitations to be considered. First, the study was observational and, thus, it was possible to detect associations but not causality. Second, due to logistic reasons, data on UO were collected only during the ICU stay; thus the study may have underestimated the incidence of AKI slightly, and detection bias may occur. However, it is plausible that the incidence of AKI is highest during the early postoperative period. Furthermore, analyses after exclusion of patients who 
received their UO-AKI diagnosis after 24 postoperative hours revealed that UO-AKI diagnosed within 24 hours especially was associated with mortality, and the potential detection bias would not change the association of only UO-AKI and higher mortality. Third, as recommended, ${ }^{13}$ the authors estimated the preceding creatinine value according to the MDRD formula in $30 \%$ of patients due to missing previous creatinine values older than 1 week. MDRD estimation was unreliable in a large retrospective study using only the KDIGO creatinine criterion. ${ }^{28}$ However, in this study's sensitivity analysis after exclusion of patients with an estimated baseline creatinine value, the results remained the same. Furthermore, the average estimated baseline glomerular filtration rate using the MDRD formula for those having a baseline creatinine value available was very close to $75 \mathrm{~mL} / \mathrm{min} / 1.73 \mathrm{~m}^{2}$. Fourth, as suggested for ICU patients, a baseline creatinine value that was older than 7 days was used as was a changing baseline during the ICU stay to cover the change within 48 hours, consistent with KDIGO stages. Both of these methods may overdiagnose Crea-AKI because of factors such as angiography-induced preoperative creatinine rise or postoperative hemodilution. This might be why Crea-AKI was not associated with a higher mortality as was UO-AKI. Fifth, only coronary artery bypass grafting patients were enrolled in the study after January 31, 2012, which, however, did not affect the incidence of AKI. In addition, in the sensitivity analyses excluding patients with chronic kidney disease and patients enrolled after January 31, the results remained significant, except in the Crea-AKI group after adjusting for risk factors. Sixth, off-pump patients were included in the study, but they comprised only $9 \%(60 / 638)$ of all patients, and exclusion of these patients did not change the results.

In summary, nearly one-third of cardiac surgical patients developed AKI using the complete KDIGO criteria. Of note, AKI patients diagnosed using only the UO criterion experienced higher crude and adjusted 2.5-year mortality compared with patients without AKI. In addition, AKI diagnosed using any of the diagnostic criteria was associated with mortality during the 2.5-year follow-up independently of EuroSCORE II. These findings corroborate the importance of $\mathrm{UO}$ in diagnosing AKI in cardiac surgical patients and the independent role of AKI contributing to mortality after cardiac surgery.

\section{Acknowledgments}

The authors acknowledge the Academy of Finland, Sigrid Juselius Foundation, and Helsinki University Central Hospital EVO Grants TYH 2011210, TYH 2013343, TYH 2013106, and T102010070. The authors thank Laura Nurminen, Sari Sutinen, Leena Pettilä, Helinä Laitinen, Heidi Syrjä, Kirsi Henttonen, and Elina Lappi in Meilahti ICUs and Kristian Andsten for their help with the Picis and Weblab databases. The authors also thank Tieto Healthcare \& Welfare for database management.

\section{Appendix A. Supplementary material}

Supplementary data associated with this article can be found in the online version at http://dx.doi.org/10.1053/j.jvca.2016.08.026.

\section{References}

1 Chertow GM, Burdick E, Honour M, et al. Acute kidney injury, mortality, length of stay, and costs in hospitalized patients. J Am Soc Nephrol 2005; $16: 3365-70$.

2 Parida S, Badhe AS. Cardiac surgery-associated acute kidney injury. J Anesth 2013;27:433-46.

3 KDIGO Work Group. KDIGO Clinical Practice Guideline for Acute Kidney Injury. Kidney Int Suppl 2012;2:19-36.

4 Kuitunen A, Vento A, Suojaranta-Ylinen R, et al. Acute renal failure after cardiac surgery: Evaluation of the RIFLE classification. Ann Thorac Surg 2006;81:542-6.

5 Lagny MG, Jouret F, Koch JN, et al. Incidence and outcomes of acute kidney injury after cardiac surgery using either criteria of the RIFLE classification. BMC Nephrol 2015;16:76.

6 McIlroy DR, Argenziano M, Farkas D, et al. Incorporating oliguria into the diagnostic criteria for acute kidney injury after on-pump cardiac surgery: Impact on incidence and outcomes. J Cardiothorac Vasc Anesth 2013;27:1145-52.

7 Hoste EAJ, Kellum JA. Acute kidney injury: Epidemiology and diagnostic criteria. Curr Opin Crit Care 2006;12:531-7.

8 Joannidis M. Classification of acute kidney injury: Are we there yet? Intensive Care Med 2007;33:572-4.

9 Kellum JA, Sileanu FE, Murugan R, et al. Classifying AKI by urine output versus serum creatinine level. J Am Soc Nephrol 2015;26:2231-8.

10 Machado MN, Nakazone MA, Maia LN. Prognostic value of acute kidney injury after cardiac surgery according to Kidney Disease: Improving Global Outcomes Definition and staging (KDIGO) criteria. PLoS One 2014;9:e98028.

11 Elmistekawy E, McDonald B, Hudson C, et al. Clinical impact of mild acute kidney injury after cardiac surgery. Ann Thorac Surg 2014;98: 815-22.

12 Nisula S, Kaukonen KM, Vaara ST, et al. Incidence, risk factors and 90day mortality of patients with acute kidney injury in Finnish intensive care units: The FINNAKI study. Intensive Care Med 2013;39:420-8.

13 Bellomo R, Ronco C, Kellum JA, et al. Acute renal failure - definition, outcome measures, animal models, fluid therapy and information technology needs: The Second International Consensus Conference of the Acute Dialysis Quality Initiative (ADQI) Group. Crit Care 2004;8:R204-12.

14 Patel BM, Chittock DR, Russell JA, et al. Beneficial effects of short-term vasopressin infusion during severe septic shock. Anesthesiology 2002;96: 576-82.

15 Russell JA, Walley KR, Singer J, et al. Vasopressin versus norepinephrine infusion in patients with septic shock. N Engl J Med 2008;358:877-87.

16 Dunser MW, Ruokonen E, Pettila V, et al. Association of arterial blood pressure and vasopressor load with septic shock mortality: A post hoc analysis of a multicenter trial. Crit Care 2009;13:R181.

17 Saravanan S, Kocarev M, Wilson RC, et al. Equivalent dose of ephedrine and phenylephrine in the prevention of post-spinal hypotension in Caesarean section. Br J Anaesth 2006;96:95-9.

18 Kaukonen KM, Vaara ST, Pettilä V, et al. Age of red blood cells and outcome in acute kidney injury. Crit Care 2013;17:R222.

19 Petäjä L, Røsjø H, Mildh L, et al. Predictive value of high-sensitivity troponin $\mathrm{T}$ in addition to EuroSCORE II in cardiac surgery. Interact Cardiovasc Thorac Surg 2016;23:133-41.

20 Kiers HD, van den Boogaard M, Schoenmakers MCJ, et al. Comparison and clinical suitability of eight prediction models for cardiac surgeryrelated acute kidney injury. Nephrol Dial Transplant 2013;28:345-51.

21 Biondi-Zoccai G, Romagnoli E, Agostoni P, et al. Are propensity scores really superior to standard multivariable analysis? Contemp Clin Trials 2011;32:731-40. 
22 Thakar CV. Perioperative acute kidney injury. Adv Chronic Kidney Dis 2013;20:67-75.

23 Oka Y, Wakayama S, Oyama T, et al. Cortisol and antidiuretic hormone responses to stress in cardiac surgical patients. Can Anaesth Soc 1981;28:334-8.

24 Kandler K, Jensen ME, Nilsson JC, et al. Acute kidney injury is independently associated with higher mortality after cardiac surgery. J Cardiothorac Vasc Anesth 2014;28:1448-52.

25 Karkouti K. Transfusion and risk of acute kidney injury in cardiac surgery. Br J Anaesth 2012;109:129-38.
26 Lassnigg A, Donner E, Grubhofer G, et al. Lack of renoprotective effects of dopamine and furosemide during cardiac surgery. J Am Soc Nephrology 2000;11:97-104.

27 Bouchard J, Soroko SB, Chertow GM, et al. Fluid accumulation, survival and recovery of kidney function in critically ill patients with acute kidney injury. Kidney Int 2009;76:422-7.

28 Bernardi MH, Schmidlin D, Ristl R, et al. Serum creatinine back-estimation in cardiac surgery patients: Misclassification of AKI using existing formulae and data-driven model. Clin J Am Soc Nephrol 2016;11:395-404. 